Published Online 2012 July 02.

Case Report

\title{
Psychogenic Vomiting Considered as Co-Morbidity With Tuberculosis in Adolescents
}

\author{
Alireza Fahimzad ${ }^{1,{ }^{*}}$, Azadeh Gharooei ${ }^{2}$, Nava Safaei ${ }^{2}$ \\ ${ }^{1}$ Pediatric Infectious Research Center (PIRC), Shahid Beheshti University of Medical Sciences, Tehran, IR Iran \\ 2 Medical Faculty, Shahid Beheshti University of Medical Sciences, Tehran, IR Iran \\ *Corresponding author: Alireza Fahimzad, Pediatric Infectious Research Center (PIRC), Shahid Beheshti University of Medical Sciences, Tehran, IR Iran. Tel.: +98-2122226941, +98-9121344595, \\ Fax:+98-2122226941, E-mail: safahimzad@yahoo.com.
}

Received: May 07, 2012; Revised: May 17, 2012; Accepted: Jun 01, 2012

\begin{abstract}
A twelve-year-old girl with disseminated Tuberculosis was complicated by psychogenic vomiting and multiple drugs' adverse effects. She was admitted to our hospital, complaining of fever, cough and abdominal pain. Diagnosis of TB was obtained by abdominal CT-scan and open liver biopsy. During the anti-TB treatment, hepatitis, hemolytic anemia and pancreatitis occurred due to some drugs' side effects In addition, her main complaint was of persistent nausea which made all probable somatic basis ruled out, since she did not also show response to routine antiemetic treatment. As a result, the patient's depression as well as the appropriate response to anti-depressant therapy helped us in confirming the diagnosis of psychogenic vomiting.
\end{abstract}

Keywords: Tuberculosis; Adolescent; Psychogenic

\section{Introduction}

Miliary Tuberculosis refers to a clinical disease resulting from uncontrolled hematogenous dissemination of mycobacterium tuberculosis (1). The term miliary TB is now used to donate all forms of progressive, widely disseminated hematogenous tuberculosis, even if the clinicopathologic or radiologic findings are absent (2). Worldwide, TB is responsible for approximately two million deaths a year (3). In past, it was widely believed that children somewhat contributed to the overall burden of tuberculosis. However, from the eight million new cases of TB reported globally in 2000, an estimated $11 \%$ occurred in children (4). Our case is a twelve-year-old girl with the diagnosis of disseminated TB, characterized with fever and abdominal pain; persistent vomiting with many different complications such as pancreatitis, gastritis, hepatitis and hemolytic anemia, which made the disease unusual and reportable.

\section{Case Presentation}

A twelve-year-old girl was admitted to our hospital with a twenty-day history of fever, cough and abdominal pain. Abdominal CT-scan - taken at the local hospital - reported multiple hypodense liver lesions. Hence, she was referred to our hospital for further studies (Figure 1).

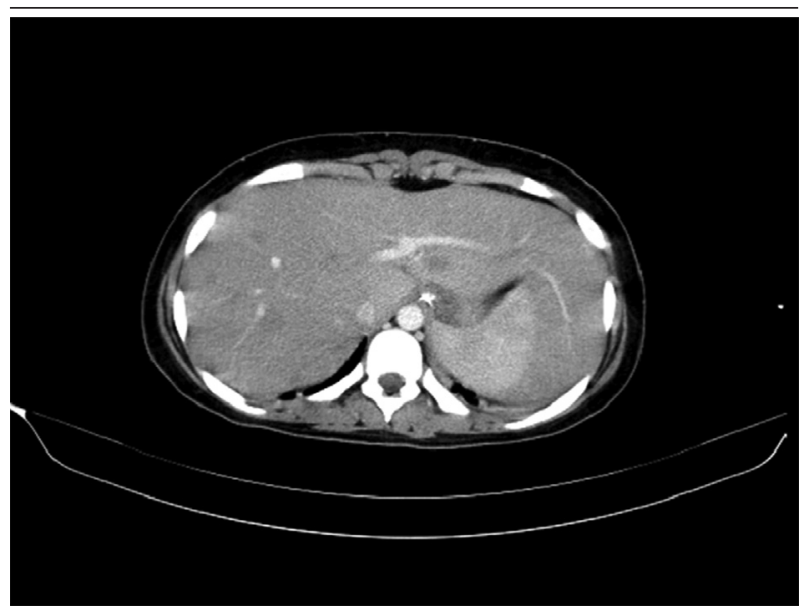

Figure 1. Abdominal CT-Scan Multiple Hypodense Liver Lesions

Clinical examination was normal when the patients admitted to the hospital. The routine hematology and biochemistry blood tests revealed microcytic hypochromic anemia and negative elevated ESR and CRP.

\subsection{Serology Test for HIV}

The initial treatment with the impression of liver abscess was done with Vancomycin, Metronidazole and Cef-

Implication for health policy/practice/research/medical education: This study is conducted for medical education purposes.

Copyright (C) 2012, Shahid Beheshti University of Medical Sciencces;. This is an Open Access article distributed under the terms of the Creative Commons Attribution License (http://creativecommons.org/licenses/by/3.0), which permits unrestricted use, distribution, and reproduction in any medium, provided the original work is properly cited. 
triaxone. Imaging confirmed liver lesions in the previous thorachoabdominal CT-scan and also showed bilateral multiple subpleural nodules. Bone marrow aspiration was normal. The clinical diagnosis was made on open liver biopsy which indicated multiple granuloma formation with central necrosis (caseous formation); no acidfast bacilli were seen. Hence, the treatment was altered with anti-TB therapy, comprising Rifampin, Ethambotol, Pyrazinamide and Isoniazide. However, the PCR result for mycobacterium TB and PPD test was also proved negative. One day after the treatment inception, upper GI bleeding occurred accompanying with fever, chills, periumblical pain and agitation. There, the routine medical therapy was done. Ranitidine, Omeprazole and vitamin $\mathrm{K}$ were added to her drug regimen and the oral feeding was stopped; but her bloody/biliary vomiting continued for several days. Ondansetrone and Sucralfate were added as well; nevertheless her condition did not improve. Transient hyperuricemia was seen, due to possible dehydration. Blood test for suspected pancreatitis was taken. The result revealed rising of amylase and lipase while an abdominal CT-scan with contrast did not show any suggestive clues of pancreatitis. Notably, upper endoscopy was not recommended due to the patient's recent surgery

The patient's condition improved gradually with conservative therapy. Ten days after anti-TB therapy, the patient's sclera became icteric with liver enzymes rising greater than three times. Accordingly Rifampin was discontinued immediately. After three days AST and ALT levels decreased and Rifampin was prescribed again but in a low dose. Considering the patient's better physical condition and normal liver enzymes, she was discharged after one month in order to continue outpatient therapy. One month after discharge from hospital, the patient readmitted with consistent biliary vomiting with four kg weight loss. During clinical examination, she was suffering from icteric sclera and pale conjunctive. Routine hematology test revealed indirect hyperbilirubinemia and anemia. Direct and indirect coombs tests were negative. Imaging showed mild hepatomegaly and mild splenomegaly in abdominal ultra-sonography. Nonetheless, brain CT-scan without contrast and chest X-ray was normal. Upper endoscopy reported severe gastritis due to regurgitation. In the end, given having no finding in our studies and according to the patient's irritability and agitation, depressed mood, decreased motivation and interest, consultation with pediatric psychiatric was done and Respiridone and Fluxetin with the impression of depressive disorder started. The patient's vomiting improved significantly and she was discharged after sixteen days with anti-depressant therapy along with two types of anti-TB drugs (Pyrazinamide and Isoniazid). The anti-TB therapy was continued for a full course of nine months.

\section{Conclusions}

With the improvement of hygiene and income level, the prevalence of TB is decreasing in developed countries although it still considers a challenge in developing countries such as Iran. The peak age of disseminated TB were children, frequently affected under the age of three, nevertheless this trend has changed over the last decade (5). Therefore, increase in the rate of HIV infection and the number of adults with impairment in cellular immunity have led to an additional peak of TB among young adults (6) Hepatic granulomas are present in more than $90 \%$ of patients with TB, approximately $70 \%$ of them have extrapulmonary TB and about $25 \%$ of them again have isolated pulmonary infections (7)., It should be borne in mind that chest radiograph allows the possibility of identifying 59 to $69 \%$ of miliary tuberculosis with high specificity and good interobserver agreement (8). In our patient there was no sign of other organ's involvement and also her chest x-ray had non-specific findings. Liver involvement in non miliary Tuberculosis is rare; and it can be presented with fever, hepatomegaly, upper abdominal pain, nausea, mild elevation of liver enzymes, low albumin, normocytic normochromic anemia and elevated ESR and is best diagnosed on liver biopsy (9).

According to the examination of thirteen cases with hepatic granuloma, it is concluded that tuberculosis is still the most common etiologic factor (10). Upper gastrointestinal bleeding and vomiting is the symptom of TB if there is a gastric involvement. Without this involvement, persistent nausea is not considered usual (11). However, there was no evidence of gastric involvement in the patient. Furthermore, elevation of amylase and lipase during the treatment made us suspicious of pancreatitis, but it was precluded after the result of the normal CTscan where she was also not responsive to conservative treatment. As several studies have documented pancreatitis in association with the use of Metronidazole, the drug-induced pancreatitis was taken into consideration although this event is rare $(12,13)$. All in all, without identifying any somatic cause for her persistent vomiting and having no response to the routine antiemetic drugs and also patient's agitation and depressed mood, psychiatric disorders have been detected. According to the study that has been done on prevalence of eating disorders, the most commonplace eating disorder and psychiatric comorbidity among children and adolescents is psychiatric nausea and the most co-morbidities are depression, intellectual disability and dissociative disorders (14).

There were two possibilities for nausea as psychosomatic disorder in our patient. First, the tuberculosis might be the etiology of this co-morbidity. In one study, $72 \%$ TB patients had severe/moderate level of anxiety and depression according to Hospital Anxiety and Depression Scale (HADS) and 22\% TB patients with co-morbid anxiety and depression showed multi drug-resistance (15). In another, it is mentioned that habitual postprandial and irregular vomiting is mostly related to major depression (16). Therefore, significant improvement in the patient's 
general condition under the anti-depressant treatment established the diagnosis. The second possibility was that antituberculose drugs induce nausea as a side effect. Among the traditional forms of first-line antituberculosis therapy, isoniazid is often associated with nervous system side effects, most prominently peripheral neuropathy, psychosis and seizures (17). Literature reviews reveal a growing number of cases of isoniazid induced psychosis. Most of the reported symptoms include paranoid delusions, visual and auditory hallucinations, suicidality, irritable mood, and disorientation (18). Our patient stayed in isoniazid therapy and never stopped it.

Our case was intertwined with other symptoms such as icter and indirect hyperbilirubinemia as a result of hemolytic anemia. Adverse effects linked with the administration of Rifampin include gastrointestinal, central nervous system, dermatologic and hematologic (thrombocytopenia and acute hemolytic anemia) reaction (19, 20). Although coincidence of different complications of a specific drug during treatment is unusual, our patient experienced some, such as hepatitis and hemolytic anemia. After excluding Rifampin from the patient's drug regimen, the hemolytic anemia ameliorated after several days. All in all, it can be concluded that apart from drugs' side effects, psychiatric disorders have a role to play specifically in adolescents, as they can cause non-negligible somatic signs.

\section{Acknowledgements}

The authors would like to thank the nurses of Mofid Children Hospital for their help.

\section{Authors' Contribution}

Dr Fahimzad dealt with treatment of the case and also revised the manuscript. Dr Gharooei and Dr Safaei prepared the manuscript.

\section{Financial Disclosure}

The authors declare that there are no conflicts of interest.

\section{Funding/Support}

The authors state that there was no financial support to the study.

\section{References}

1. Sharma SK, Mohan A, Sharma A, Mitra DK. Miliary tuberculosis: new insights into an old disease. Lancet Infect Dis. 2005;5(7):415-30.

2. Mert A, Bilir M, Tabak F, Ozaras R, Ozturk R, Senturk H, et al. Miliary tuberculosis: clinical manifestations, diagnosis and outcome in 38 adults. Respirology. 2001;6(3):217-24.

3. Corbett EL, Watt CJ, Walker N, Maher D, Williams BG, Raviglione MC, et al. The growing burden of tuberculosis: global trends and interactions with the HIV epidemic. Arch Intern Med. 2003;163(9):1009-21.

4. Nelson LJ, Wells CD. Global epidemiology of childhood tuberculosis. Int J Tuberc Lung Dis. 2004;8(5):636-47.

5. Smith S, Jacobs RF, Wilson CB. Immunobiology of childhood tuberculosis: A window on the ontogeny of cellular immunity. J Pediatr.1997;131(1):16-26.

6. Braun MM, Cote TR, Rabkin CS. Trends in death with tuberculosis during the AIDS era. JAMA. 1993;269(22):2865-8.

7. Klatskin G. Hepatic granulomata: problems in interpretation. $M t$ Sinai J Med. 1977;44(6):798-812.

8. Kwong JS, Carignan S, Kang EY, Muller NL, FitzGerald JM. Miliary tuberculosis. Diagnostic accuracy of chest radiography. Chest. 1996;110(2):339-42.

9. Desai CS, Josh AG, Abraham P, Desai DC, Deshpande RB, Bhaduri A, et al. Hepatic tuberculosis in absence of disseminated abdominal tuberculosis. Ann Hepatol. 2006;5(1):41-3.

10. Onal IK, Ersoy O, Aydinli M, Yonem O, Harmanci O, Sokmensuer C, et al. Hepatic granuloma in Turkish adults: a report of 13 cases. EurJ Intern Med. 2008;19(7):527-30.

11. Rao YG, Pande GK, Sahni P, Chattopadhyay TK. Gastroduodenal tuberculosis management guidelines, based on a large experience and a review of the literature. Can J Surg. 2004;47(5):364-8.

12. Celifarco A, Warschauer C, Burakoff R. Metronidazole-induced pancreatitis. Am J Gastroenterol.1989;84(8):958-60.

13. Sanford KA, Mayle JE, Dean HA, Greenbaum DS. Metronidazoleassociated pancreatitis. Ann Intern Med.1988;109(9):756-7.

14. Mammen P, Russell S, Russell PS. Prevalence of eating disorders and psychiatric comorbidity among children and adolescents. Indian Pediatr. 2007;44(5):357-9.

15. Aamir S A. Co-morbid anxiety and depression among pulmonary tuberculosis patients. J Coll Physicians Surg Pak. 2010;20(10):703-4.

16. Muraoka M, Mine K, Matsumoto K, Nakai Y, Nakagawa T. Psychogenic vomiting: the relation between patterns of vomiting and psychiatric diagnoses. Gut.1990;31(5):526-8.

17. Kass JS, Shandera WX. Nervous system effects of antituberculosis therapy. CNS Drugs. 2010;24(8):655-67.

18. Witkowski AE, Manabat CG, Bourgeois JA. Isoniazid-associated psychosis. Gen Hosp Psychiatry. 2007;29(1):85-6.

19. Martinez E, Collazos J, Mayo J. Hypersensitivity reactions to rifampin. Pathogenetic mechanisms, clinical manifestations, management strategies, and review of the anaphylactic-like reactions. Medicine (Baltimore). 1999;78(6):361-9.

20. Neunert CE, Paranjape GS, Cameron S, Rogers ZR. Intravascular hemolysis following low dose daily rifampin. Pediatr Blood Cancer. $2008 ; 51(6): 821-3$. 